\title{
A Functional Screen to Characterize the Secretomes of Eukaryotic Pathogens and Their Hosts In Planta
}

\author{
Sang-Jik Lee, ${ }^{1}$ Brendan S. Kelley, ${ }^{1}$ Cynthia M. B. Damasceno, ${ }^{1}$ Bonnie St. John, ${ }^{1}$ Byung-Soo Kim, ${ }^{2}$ \\ Byung-Dong Kim, ${ }^{3}$ and Jocelyn K. C. Rose ${ }^{1}$ \\ ${ }^{1}$ Department of Plant Biology, Cornell University, Ithaca, NY 14853, U.S.A.; ${ }^{2}$ Department of Horticulture, Kyungpook \\ National University, Daegu 702-701, Korea; ${ }^{3}$ Center for Plant Molecular Genetics and Breeding Research, Seoul National \\ University, Seoul 151-921, Korea
}

Submitted 24 May 2006. Accepted 26 July 2006.

\begin{abstract}
Complex suites of proteins that are secreted by plants and phytopathogens into the plant apoplast play crucial roles in surveillance, assault, defense, and counter-defense. Highthroughput genome-scale strategies are being developed to better understand the nature of these "secretomes" and the identity of pathogen-derived effector proteins that subvert plant defenses and promote pathogenicity. Although combined bioinformatic and experimental approaches recently have provided comprehensive coverage of secreted proteins from bacterial phytopathogens, far less is known about the secretomes and batteries of effectors of eukaryotic phytopathogens; notably fungi and oomycetes. The yeast secretion trap (YST) represents a potentially valuable technique to simultaneously target pathogen and host secretomes in infected plant material. A YST screen, using a new vector system, was applied to study the interaction between tomato (Solanum lycopersicum) and the oomycete Phytophthora infestans, revealing sets of genes encoding secreted proteins from both pathogen and host. Most of those from the oomycete had no identifiable function and were detectable in planta only during pathogenesis, underlining the value of YST as a tool to identify new candidate effectors and pathogenicity factors. In addition, the majority of the $P$. infestans proteins had homologs in the genomes of the related oomycetes $P$. sojae and $P$. ramorum.
\end{abstract}

Additional keywords: cell wall, signal peptide.

The plant cell wall represents the interface between the plant and the abiotic and biotic environments and provides a crucial defensive barrier against microbial pathogens that constantly seek to invade and exploit a new host. Therefore, the plant wall and apoplastic milieu can be viewed as a molecular battleground, a key element of which involves the co-evolution and interplay between plant- and pathogen-secreted proteomes, or secretomes, that are required for surveillance, assault, defense, and counterdefense.

Plant cells secrete a broad spectrum of constitutively expressed and inducible defense-related proteins and peptides whose functions include degradation of microbial cell wall polymers and the inhibition of proteins secreted by the patho-

Corresponding author: Jocelyn K. C. Rose; Telephone: +1.607.255.4781; Fax: +1.607.255.5407; E-mail: jr286@ cornell.edu

Nucleotide sequence information is available under accession numbers: CV970753-CV970774, CV970777, and CV970779-CV970800. gen (van der Hoorn and Jones 2004). Similarly, phytopathogens secrete suites of peptides and proteins during infection which can remain associated with the hyphal wall (Rast et al. 2003) or which are more mobile in the plant apoplast. Many of these proteins are broadly referred to as "effectors," implying pathogen-derived molecules that alter host cell structure or function and influence pathogenicity or defense responses. They include enzymes involved in disassembly of the plant wall (Huitema et al. 2004; Vorwerk et al. 2004) and suppressors of extracellular plant defenses (Abramovitch and Martin 2004; Huitema et al. 2004; Rose et al. 2002). Depending on the nature of the interacting host and pathogen, specific secreted proteins can interact with the product of a corresponding host resistance $(R)$ gene, in which case they are termed avirulence (Avr) proteins, and this interaction results in disease resistance (Dangl and Jones 2001), culminating in a hypersensitive response (HR).

As well as secreting proteins into the apoplast, gram-negative bacteria have evolved a type III secretion system (TTSS) to translocate virulence proteins in a specialized structure across the host plasma membrane and cell wall and inject them directly into the host cytosol (Alfano and Collmer 2004; Guttman et al. 2002). The translocated proteins, which can act as virulence and avirulence factors, also are termed effectors, playing a pivotal role in pathogenicity by suppressing or inducing defense responses such as the HR (Abramovitch and Martin 2004; Alfano and Collmer 2004; Chang et al. 2004). The identification of bacterial Avr proteins and effectors has been accelerated dramatically in the last few years by the combined use of computational and experimental strategies to characterize the TTSS-associated secretome (Chang et al. 2005; Greenberg and Vinatzer 2003). This has been particularly effective in the case of Pseudomonas syringae, resulting in essentially saturating coverage that will provide an excellent platform to determine translocated effector function, mechanism of action, and biological significance.

In contrast, far less is known about the secretome or the nature of potential effectors from eukaryotic microbial phytopathogens. For example, although most Avr proteins from eukaryotic phytopathogens that have been studied to date are apoplastic (Rep 2005), it recently has been shown that, as with bacterial phytopathogens, some Avr proteins from fungi and oomycetes are translocated into plant cells (Armstrong et al. 2005; Catanzariti et al. 2006; Rehmany et al. 2005). This new discovery further illustrates the complexity of the secretomes of plant pathogens and their hosts and, although individual components of these extracellular systems are being revealed through specific assays or molecular screens, progress is incre- 
mental and, clearly, much remains to be discovered. By analogy to the remarkable progress that has been made with profiling the TTSS secretome, a more extensive catalog of the secretomes of a eukaryotic phytopathogen-plant interaction would provide valuable new insights into apoplastic processes and the extent and nature of the suites of secreted effectors.

To date, the only reported large-scale secretome screen for a eukaryotic plant pathogen was a computational prediction of secreted proteins based on 1,000 unigenes from the oomycete Phytophthora infestans (Torto et al. 2003). As with the recent high-resolution studies of the bacterial TTSS secretome, an equivalent experimental screen for secreted proteins would provide an important complement to such a bioinformatic approach. In this regard, the yeast secretion trap (YST) functional screen represents an attractive option (Jacobs et al. 1997; Lee et al. 2004). This technique involves ligating a pool of heterologous cDNAs derived from infected plant tissue to a yeast invertase gene (suc2) and transforming the resulting library into an invertase-deficient yeast mutant, which then is grown on a medium with sucrose as the sole carbon source. Any yeast transformant containing a heterologous cDNA that directs secretion of the recombinant invertase fusion protein has the potential to rescue of the mutant. The resulting yeast transformants then can be isolated and the gene encoding the secreted protein identified.

This article describes the development and application of a new modified YST vector system (pYST-0 to pYST-2) to simultaneously identify secreted proteins involved in the interaction between tomato (Solanum lycopersicum) and the oomycete $P$. infestans. The temporal expression patterns of the resulting $P$. infestans genes are reported, together with detection of orthologous genes in the recently completed $P$. sojae and $P$. ramorum genome sequences.

\section{RESULTS}

\section{Development of a pYST vector set and validation with plant pathogen-secreted proteins.}

It has been noted in several studies using the YST that the design of the cloning vector severely limits the number of cDNAs that potentially can be identified, given that it always has been used in a single vector system. Even though the anchored random primer used in the screen theoretically should anneal randomly to the transcripts and, thus, in all three reading frames, in practice the pYST one-vector system does not appear to provide full coverage. Indeed, the limited size of a one-vector-derived library suggests that priming does not occur randomly. If this is the case, there is as low as a one-in-three probability that a particular cDNA encoding a secreted protein will be ligated in frame with invertase and the necessary fusion protein secreted. Using the pSMASH vector (Goo et al. 1999) as a template, the pYST three-vector system was developed (pYST-0, pYST-1, and pYST-2) to allow cloning of any signal peptide (SP)-encoding cDNA to generate an in-frame fusion with the invertase, regardless of the reading frame at the $3^{\prime}$ end of the heterologous cDNA and to compensate for any potential nonrandom annealing of the anchored random primer (Fig. 1A and B). We observed that the YST clones identified through the screen described here were present in equal proportions in the three YST vectors, thereby confirming the value of a threevector set.

It also was noted that, when pSMASH was used in preliminary screens, yeast transformants were consistently recovered containing the $s u c 2$ gene, with no additional heterologous sequence as a fusion (data not shown). The first encoded amino acid of the truncated suc2 invertase without the SP is a methionine $\left(\mathrm{Met}^{21}\right)$, and it was hypothesized that this might give rise to an artifactual "SP-like" sequence, leading to a low-level secretion of the invertase protein alone and, consequently, false positives. A previous study using pSMASH to identify Arabidopsis thaliana-secreted proteins reported that $24 \%$ of the cDNA clones were false positives (Goo et al. 1999) and a similar proportion (17\% of isolated clones) was described when using related YST vectors that contain $\mathrm{Met}^{21}$ and $\mathrm{Thr}^{22}$ (Jacobs et al. 1997). Accordingly, the YST vector set was redesigned to eliminate the first two amino acids $\left(\mathrm{Met}^{21}\right.$ and $\mathrm{Thr}^{22}$ ) of the mature protein; however, the frequency of false positives containing no heterologous insert using one vector of the threevector YST alone was the same as that using the original pSMASH vector. When the pYST three-vector system was used in the current study, the frequency of such false positives was greatly reduced and only 5 of 157 cDNAs sequenced contained no heterologous sequence. Therefore, this increased efficiency was likely a consequence of the more effective capture of cDNAs in all reading frames, resulting in a higher ratio of SP-encoding sequences to false positives.

A single-vector YST system recently was used to confirm the secretion of specific fungal proteins (Catanzariti et al. 2006); however, to date, it has not been applied to screen libraries of
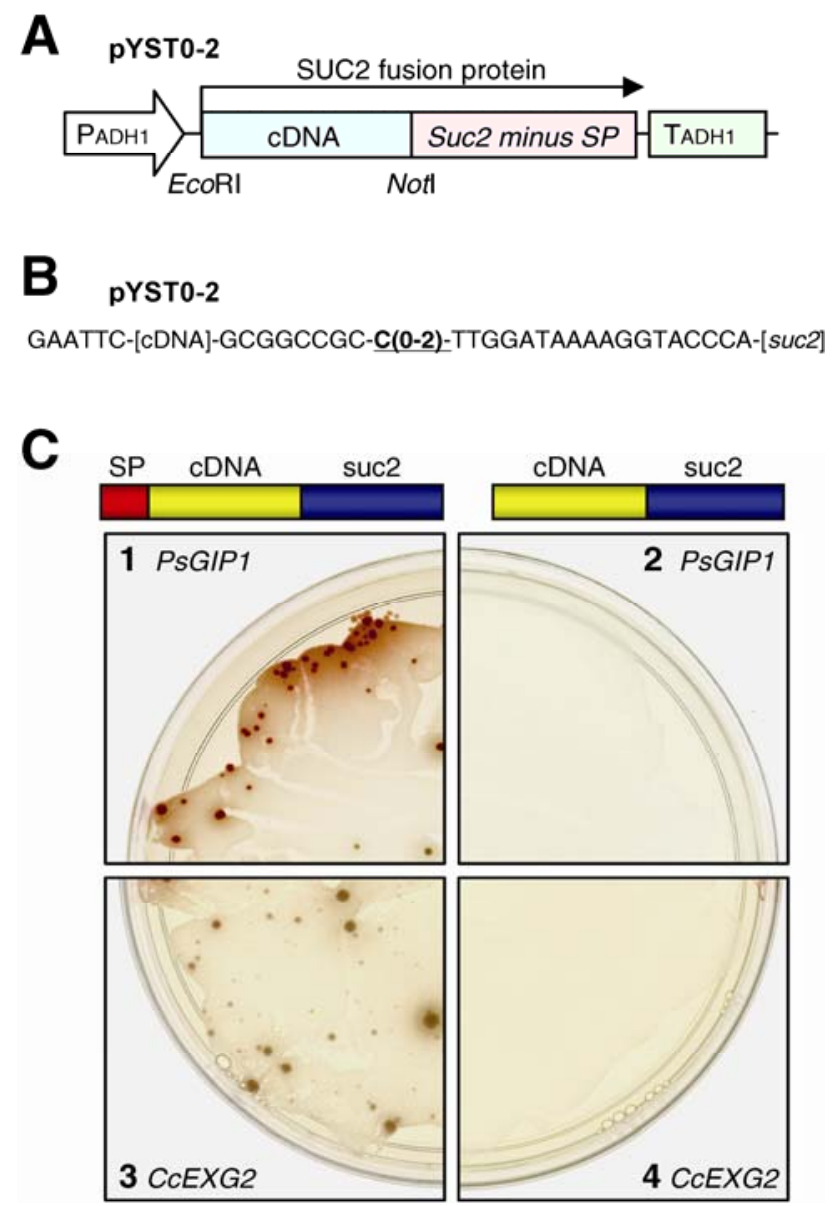

Fig. 1. Modified yeast secretion trap (pYST) cDNA library vectors and their use to identify oomycete and fungal secreted proteins. A, Schematic diagram of the three YST vectors (pYST-0, pYST-1, and pYST-2) used for cDNA library construction. Restriction enzyme sites for cDNA insertions and the positions of the promoter (PADH1) and terminator (TADH1) of the alcohol dehydrogenase gene are indicated. suc2 represents a yeast invertase gene lacking its own signal peptide (SP) and initiator methionine. B, Nucleotide sequences of the pYST vector set. The variable $\mathrm{C}$ stretch in the pYST series of vectors is underlined. C, YST analysis of $P$. sojae GIPI and Cochliobolus carbonum EXG2 with (panels 1 and 3, respectively) or without (panels 2 and 4, respectively) their SPs. 
cDNAs from pathogen-infected plant tissues. To confirm the efficacy of the new modified pYST vector system as a means to identify secreted proteins from eukaryotic microbes, notably oomycetes and fungi, the full-length open reading frames (ORFs) of glucanase inhibitor protein 1 (PsGIP1) (Rose et al. 2002) from the oomycete $P$. sojae and exo-1,3- $\beta$-glucanase 2 from the ascomycete fungus Cochliobolus carbonum (CcEXG2) (Kim et al. 2001), two known secreted proteins, were cloned into pYST- 0 and pYST-1, respectively. Yeast transformants expressing the PsGIP1 $1^{1-257}$ :suc2 or the CcEXG2 $2^{1-}$ ${ }^{422}$ :suc2 fusions grew on sucrose, whereas yeast expressing the Ps $G I P 1^{22-257}$ or the CcEXG $2^{19-422}$ fusions, corresponding to the same proteins without the predicted SPs, did not grow (Fig. 1C). These results confirmed that the pYST vector system is a potentially valuable tool to identify new secreted proteins from oomycetous and fungal plant pathogens.

\section{Yeast secretion trap library construction}

and screening of $P$. infestans-infected tomato leaf cDNAs.

Although the experiments with PsGIP1 and CcEXG2 demonstrated that the YST system can identify pathogen-derived secreted proteins, a critical question is whether it can be used to isolate both plant- and pathogen-secreted proteins that are expressed in planta during pathogenesis. A potential obstacle is

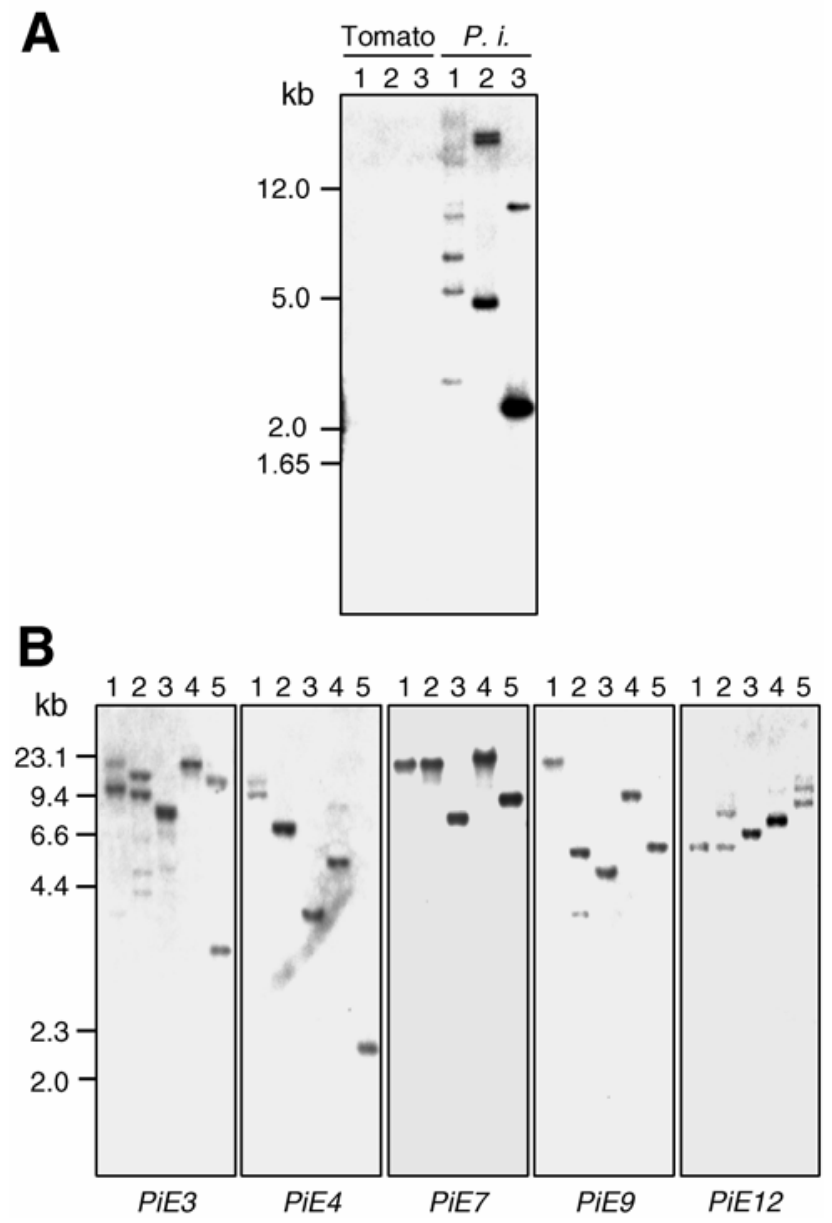

Fig. 2. Genomic DNA gel blot analysis of PiE clones. A, Representative genomic DNA gel blot screen of tomato and Phytophthora infestans (P.i.). DNA was digested with EcoRI (1), EcoRV (2), and BamHI (3) and hybridized with a $P i E 2$ probe to confirm the species of origin. B, Genomic DNA gel blot analysis of the $P$. infestans genes. Genomic $P$. infestans DNA $(10 \mu \mathrm{g})$ was digested with EcoRI (1), EcoRV (2), SacI (3), XbaI (4), and BamHI (5) and hybridized with the indicated PiE clones. Molecular size markers are indicated $(\mathrm{kb})$. the high ratio of plant to microbial biomass, where the greater abundance of plant host cDNAs encoding secreted proteins might make the detection of pathogen-derived cDNAs relatively rare. A small-scale pilot study was performed using the pYST vector set to screen cDNAs derived from tomato leaves that were infected with a tomato-specialized isolate of the oomycete pathogen $P$. infestans, which resulted in a compatible interaction. To reduce the need to resequence redundant clones, heterologous cDNAs in the YST vectors derived from the first set of approximately 50 yeast colonies were used to screen subsequent sets of selected colonies by Southern blot analysis of the polymerase-chain reaction (PCR)-amplified inserts, in an iterative process. Using this approach, 625 separate yeast transformants were screened and 157 plasmids sequenced, resulting in the identification of 45 unique YST cDNAs, with an average insert size of approximately $400 \mathrm{bp}$.

To determine the species of origin of these clones, the sequences were used to search a number of DNA databases, taking advantage of the substantial expressed sequence tag (EST) collections for both tomato and $P$. infestans. In most cases, the cDNAs corresponded to a previously submitted EST, whereas others were new sequences. The percentage of GC content can be markedly different between tomato and Phytophthora spp. (Qutob et al. 2000) and, in theory, could be used to distinguish between the plant- and oomycete-derived genes. However, this did not always provide a clear indication; therefore, tomato or $P$. infestans genomic Southern blot analyses were performed using each cDNA that was not present in the databases. This proved to be definitive in all cases, and representative results are shown in Figure $2 \mathrm{~A}$ and $\mathrm{B}$, indicating that PiE2 (P. infestans Extracellular 2) (Table 1) cross-hybridizes only with $P$. infestans genomic DNA and showing the genomic organization of the genes encoding the $P$. infestans-secreted proteins PiE3, PiE4, PiE7, PiE9, and PiE12. These clones were selected arbitrarily from among those that are listed as unknown proteins and currently have no annotation to confirm their function. Despite the difference in relative biomass, it was determined that equal numbers of unique cDNAs were derived from the plant and the pathogen (Tables 1 and 2).

\section{Classification of $P$. infestans and tomato YST clones.}

Only 6 of the 23 P. infestans YST clones could be annotated based on sequence identity or similarity to previously identified genes (Table 1). Two (PiE1 and PiE5) corresponded to the elicitins ELI-INF1 and ELI-INF5, which are members of a family of evolutionarily related proteins that recently have been reclassified (Jiang et al. 2006). PiE1 (ELI-INF1, formerly INF1) is a well-characterized secreted elicitor of the hypersensitive response (Kamoun et al. 1997). Other annotated clones included an extracellular metallopeptidase (PiE17) and a cutinase (PiE18), classes of hydrolytic enzymes that commonly are secreted by phytopathogens and are believed to be pathogenicity determinants. Another previously reported P. infestans gene that was identified through the YST screen (PiE19) was scr74, which encodes a cysteine-rich species-specific elicitor-like protein (Liu et al. 2005), with sequence similarity to $\mathrm{PcF}$, a necrosis-inducing protein secreted by $P$. cactorum (Orsomando et al. 2001) and a candidate $a v r$ gene $\operatorname{scr} 91$ (Bos et al. 2003). Interestingly, the last annotated $P$. infestans YST clone (PiE16) has $46 \%$ amino acid sequence identity with a recently identified protein pheromone, termed temptin, from the sea hare Aplysia brasiliana (Cummins et al. 2004). Among the $23 \mathrm{PiE}$ clones, all but 8 had homologs in the $P$. sojae and $P$. ramorum genomes.

Approximately half of the YST clones originating from tomato (Table 2, SIE, S. lycopersicum Extracellular) have an association with plant defenses, including previously reported 
pathogenesis-related (PR) proteins (e.g., SlE3 and SlE17), structural proteins such as extensin and related glycine-rich proteins, glycosyl hydrolases that target glycans that are typical constituents of microbial cell walls (e.g., SlE2 and SlE16), and a putative peroxidase (SlE15). Other YST clones are pre- dicted to encode proteins whose defensive role is less well defined, such as stellacyanin (SlE11), but that have been reported to be induced by wounding or elicitors (Takemoto et al. 2003). A third of the clones (SlE4, SlE9, SlE13, and SlE20-22) had no clear homology to known genes or obvious functional domains

Table 1. Phytophthora infestans yeast secretion trap clones isolated from P. infestans-infected tomato leaves ${ }^{\mathrm{a}}$

\begin{tabular}{|c|c|c|c|c|c|c|c|c|c|}
\hline \multirow[b]{2}{*}{ Clone (acc. no.) ${ }^{b}$} & \multicolumn{4}{|c|}{ Database annotation } & \multicolumn{2}{|c|}{ Homologue } & \multirow{2}{*}{$\frac{\text { PexFinder }}{\text { HMM }}$} & \multicolumn{2}{|c|}{ SignalP 3.0 score } \\
\hline & Protein name & GenBank $^{c}$ & E value $^{d}$ & Species & P. sojae & P. ramorum & & HМM & $\mathbf{N N}$ \\
\hline PiE1 (CV970777) & Elicitin ELI-INF1 & AAB49807 & $1 e-34$ & P. infestans & 128080 & 78569 & 1.000 & 1.000 & 0.783 \\
\hline PiE2 (CV970779) & Unknown protein & CV933463 & $1 e-58$ & P. infestans & 134030 & 80982 & $0.134^{(\mathrm{SA})}$ & $0.002^{(\mathrm{SA})}$ & 0.168 \\
\hline PiE3 (CV970780) & Unknown protein & CV947495 & $2 \mathrm{e}-154$ & P. infestans & $\ldots$ & $\ldots$ & 0.999 & 1.000 & 0.845 \\
\hline PiE4 (CV970781) & Unknown protein & CV945595 & $3 e-100$ & P. infestans & 130440 & 81685 & 0.997 & 0.999 & 0.785 \\
\hline PiE5 (CV970782) & Elicitin ELI-INF5 & AAL16012 & $3 e-29$ & P. infestans & 108238 & 71633 & 0.997 & 0.997 & 0.642 \\
\hline PiE6 (CV970783) & Unknown protein & AF424687 & $0.0(\mathrm{n})$ & P. infestans & 136058 & 81781 & 1.000 & 1.000 & 0.775 \\
\hline PiE7 (CV970784) & Unknown protein & CV969546 & 0 & P. infestans & 144427 & 78163 & 0.974 & 0.96 & 0.623 \\
\hline PiE8 (CV970785) & Unknown protein & CV953356 & $7 e-104$ & P. infestans & $\ldots$ & $\ldots$ & $0.106^{(\mathrm{SA})}$ & $0.078^{(\mathrm{SA})}$ & 0.686 \\
\hline PiE9 (CV970786) & Unknown protein & CV946996 & 0 & P. infestans & $\ldots$ & $\ldots$ & 0.995 & 0.996 & 0.623 \\
\hline PiE10 (CV970787) & Hypothetical protein & $\ldots$ & $\ldots$ & $\ldots$ & 141791 & 82244 & 1.000 & 1.000 & 0.85 \\
\hline PiE11 (CV970788) & Unknown protein & XP_370456 & $6 e-11$ & Magnaporthe grisea & $\ldots$ & $\ldots$ & 0.998 & 0.997 & 0.745 \\
\hline PiE12 (CV970789) & Unknown protein & CV900695 & $0.0(\mathrm{n})$ & P. infestans & 135684 & 82920 & 0.996 & 0.996 & 0.708 \\
\hline PiE13 (CV970790) & Unknown protein & CV899036 & $0.0(\mathrm{n})$ & P. infestans & $\ldots$ & $\ldots$ & 0.993 & 0.994 & 0.754 \\
\hline PiE14 (CV970791) & Unknown protein & CV918188 & $1 e-156$ & P. infestans & 135689 & 86904 & 0.996 & 0.996 & 0.671 \\
\hline PiE15 (CV970792) & Unknown protein & XP_363174 & $3 e-04$ & M. grisea & $\ldots$ & $\ldots$ & 0.999 & 0.999 & 0.72 \\
\hline PiE16 (CV970793) & Temptin & AAS 92605 & $1 e-16$ & Aplysia brasiliana & 143786 & 78188 & 0.999 & 0.999 & 0.804 \\
\hline PiE17 (CV970794) & Metallopeptidase & NP_639017 & $7 e-16$ & Xanthomonas campestris & 139557 & 74032 & 0.982 & 0.997 & 0.742 \\
\hline PiE18 (CV970795) & Cutinase & AAO92431 & $2 e-50$ & P. brassicae & 123647 & 39832 & 0.999 & 0.998 & 0.824 \\
\hline PiE19 (CV970796) & Elicitor-like SCR74 & AAU21458 & $3 e-37$ & P. infestans & $\ldots$ & 43241 & 0.997 & 0.996 & 0.776 \\
\hline PiE20 (CV970797) & Unknown protein & CV965926 & $4 \mathrm{e}-140$ & P. infestans & $\ldots$ & $\ldots$ & 1.000 & 1.000 & 0.773 \\
\hline PiE21 (CV970798) & Unknown protein & CV929998 & 0 & P. infestans & 121909 & 71256 & 0.968 & $0.415^{(\mathrm{SA})}$ & 0.585 \\
\hline PiE22 (CV970799) & Unknown protein & CAE67074 & $1 e-26$ & Caenorhabditis elegans & 138595 & 81609 & 1.000 & 1.000 & 0.8 \\
\hline PiE23 (CV970800) & Unknown protein & CV948862 & 0 & P. infestans & 135685 & 82921 & 0.999 & 0.986 & 0.591 \\
\hline
\end{tabular}

${ }^{a} P$. sojae and $P$. ramorum genome sequences at the JGI website; HMM and NN = hidden Markov model and neural network methods, respectively; scores in bold indicate clones with no predicted signal peptide; $(\mathrm{n})=$ nucleotide-nucleotide BLAST and $(\mathrm{SA})=$ predicted signal anchor.

b Clone name and $P$. infestans expressed sequence tag accession number.

c Accession number.

${ }^{\mathrm{d}}$ E value from translating BLAST or protein-protein BLAST.

Table 2. Tomato yeast secretion trap clones isolated from Phytophthora infestans-infected tomato leaves

\begin{tabular}{|c|c|c|c|c|c|c|c|}
\hline \multirow[b]{2}{*}{ Clone (acc. no.) ${ }^{\mathbf{b}}$} & \multicolumn{5}{|c|}{ Database annotation } & \multicolumn{2}{|c|}{ SignalP 3.0 score ${ }^{\mathrm{a}}$} \\
\hline & Protein name & SGN $^{c}$ acc. no. & GenBank acc. no. & E value $^{d}$ & Species & HMM & $\mathbf{N N}$ \\
\hline SIE1 (CV970753) & Glycine-rich protein & SGN-U220422 & AAP83840 & 0.018 & Solanum lycopersicum & 0.992 & 0.876 \\
\hline S1E2 (CV970754) & $\begin{array}{l}\text { Glycosyl hydrolase family } \\
\text { protein } 17\end{array}$ & SGN-U223104 & NP_564059 & $5 e-27$ & Arabidopsis thaliana & 1.000 & 0.875 \\
\hline SIE3 (CV970755) & $\begin{array}{l}\text { Pathogenesis-related (PR) } \\
\text { protein PR-1 }\end{array}$ & SGN-U212923 & P04284 & $4 e-39$ & S. lycopersicum & 1.000 & 0.945 \\
\hline SIE4 (CV970756) & Expressed protein & SGN-U216346 & BG127004 & $6 e-147$ & S. lycopersicum & 1.000 & 0.882 \\
\hline SIE5 (CV970757) & Extensin & SGN-U285067 & X55683 & $2 \mathrm{e}-35(\mathrm{n})$ & S. lycopersicum & 0.998 & 0.742 \\
\hline SIE6 (CV970758) & Glycine-rich protein & SGN-U217408 & CAB67122 & $2 \mathrm{e}-08$ & Nictotiana tabacum & 0.973 & 0.874 \\
\hline SIE7 (CV970759) & Glycine-rich protein & SGN-U212549 & T07013 & $5 e-09$ & S. lycopersicum & 0.997 & 0.893 \\
\hline SIE8 (CV970760) & Glycine-rich protein & $\ldots$ & P09789 & 0.27 & Petunia $\times$ hybrida & 0.993 & 0.784 \\
\hline SIE9 (CV970761) & Unknown protein & SGN-U225752 & NP_174546 & $2 \mathrm{e}-11$ & Arabidopsis thaliana & 0.000 & 0.063 \\
\hline S1E10 (CV970762) & Phosphate/PEP translocator & SGN-U214131 & T03836 & $8 e-95$ & Nicotiana tabacum & 0.006 & 0.241 \\
\hline S1E11 (CV970763) & Stellacyanin-like protein & SGN-U215639 & AAG01535 & $3 e-34$ & Capsicum anпиит & 0.989 & 0.824 \\
\hline SIE12 (CV970764) & GDSL-motif lipase & SGN-U214390 & T02229 & $7 e-45$ & N. tabacum & 1.000 & 0.909 \\
\hline S1E13 (CV970765) & Expressed protein & SGN-U213711 & AAL16012 & $\ldots$ & $\ldots$ & 0.999 & 0.89 \\
\hline S1E14 (CV970766) & Glycine-rich protein & SGN-U238191 & AAP83840 & $1 \mathrm{e}-09$ & S. lycopersicum & 0.994 & 0.905 \\
\hline S1E15 (CV970767) & Putative peroxidase & SGN-U219648 & AAK59478 & $2 \mathrm{e}-34$ & A. thaliana & 1.000 & 0.798 \\
\hline S1E16 (CV970768) & $\beta$-1,3-Glucanase-like protein & SGN-U215533 & CAB80165 & $2 \mathrm{e}-79$ & A. thaliana & 0.998 & 0.795 \\
\hline S1E17 (CV970769) & PR protein $\mathrm{P} 2$ & SGN-U214985 & CAA41439 & $3 e-57$ & S. lycopersicum & 0.994 & 0.749 \\
\hline S1E18 (CV970770) & Glycine-rich protein & SGN-U216000 & BP905591 & 0.27 & S. lycopersicum & 0.994 & 0.892 \\
\hline S1E19 (CV970771) & $\begin{array}{l}\text { Protease inhibitor/seed } \\
\text { storage/LTP family }\end{array}$ & SGN-U218425 & ВТ014512 & $3 e-30$ & S. lycopersicum & 0.997 & 0.778 \\
\hline S1E20 (CV970772) & Unknown protein & SGN-U217114 & CV966669 & $3 e-08$ & N. tabacum & 0.978 & 0.852 \\
\hline S1E21 (CV970773) & Wound-induced protein & SGN-U214651 & CAA31852 & $3 e-37$ & S. lycopersicum & 0.999 & 0.916 \\
\hline S1E22 (CV970774) & Harpin-induced protein & SGN-U217180 & NP_198513 & $5 e-32$ & A. thaliana & 0.000 & 0.02 \\
\hline
\end{tabular}

${ }^{a}$ HMM and NN = hidden Markov model and neural network methods, respectively; scores in bold indicate clones with no predicted signal peptide.

${ }^{\mathrm{b}}$ Clone name and Solanum lycopersicum expressed sequence tag accession number.

${ }^{\mathrm{c}} \mathrm{SGN}=\mathrm{SOL}$ Genomics Network hosted at Cornell University.

${ }^{\mathrm{d}} \mathrm{E}$ value from translating BLAST or protein-protein BLAST; $(\mathrm{n})=$ nucleotide-nucleotide BLAST. 
and motifs. Three clones (SlE9, SlE10, and SlE22) had no predicted SP (Table 2, bold and underlined) with either hidden Markov model (HMM) or neural network (NN) methods.

The predicted proteins derived from all the YST clones were searched for the presence of an N-terminal SP using SignalP (version 3.0) (Bendtsen et al. 2004) and Tables 1 and 2 show the scores using either an HMM or NN method (those scores indicating no predicted SP are in bold and underlined). In addition, the equivalent scores generated using an earlier version of SignalP (SignalP, v2.0.b2) that formed the basis of PexFinder, an algorithm designed to predict extracellular proteins from EST collections and previously used in an analysis of $P$. infestans ESTs (Torto et al. 2003), are shown in Table 1. Of the PiE proteins, 20 were predicted to have an N-terminal SP and 3 (PiE2, PiE8, and PiE21) an N-terminal signal anchor (SA), which is defined as an uncleaved SP. An NN D score of 0.48 or greater is an indication of the presence of an SP, and all PiE proteins had a positive score apart from PiE2. It was noted that the older version of SignalP used in PexFinder gave predictions similar to those of the newer version 3.0, with the exceptions of PiE21, which was given a high probability score by PexFinder but a low score or an indicated SA using version 3.0 (Table 1). In contrast, three SIE proteins (SIE9, SIE10, and SIE22) had no predicted SP or SA with either the NN or HMM (Table 2).

\section{Transcriptional dynamics} of $P$. infestans YST clones during infection of tomato.

A key objective of this study was to determine whether the improved YST vector system could identify new potential effectors and pathogenicity factors that are secreted specifically by eukaryotic pathogens in planta during pathogenesis. The expression patterns of a selection of the $\mathrm{PiE}$ clones were compared by Northern blot analysis of RNA from axenically

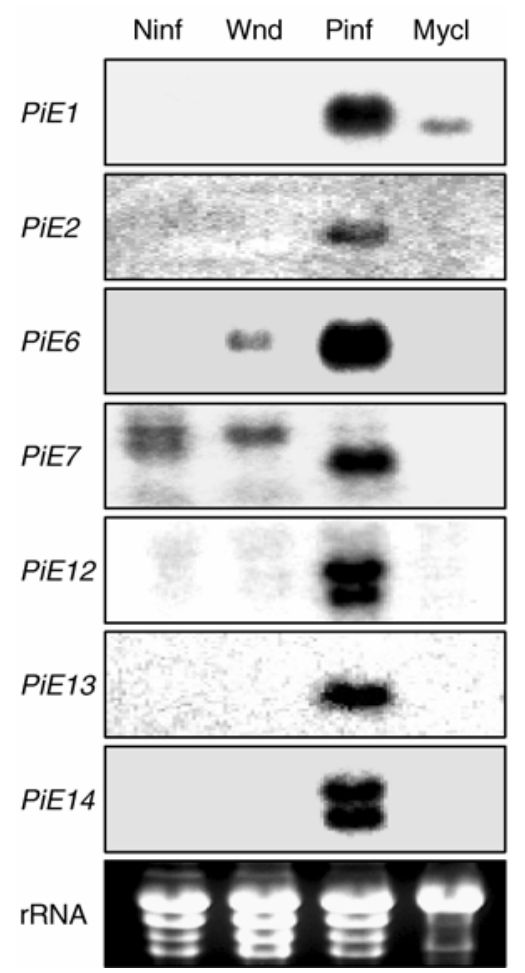

Fig. 3. RNA gel blot analysis of several PiE clones. Total RNA ( $20 \mu \mathrm{g}$ per lane) was isolated from noninfected tomato leaves (Ninf), leaves that had been wounded (Wnd) or infected with $P$. infestans (Pinf), and axenically grown $P$. infestans mycelia (Mycl). Ethidium bromide-stained rRNAs are shown to evaluate loading. grown $P$. infestans mycelia, control or wounded tomato leaves, or leaves 4 days post inoculation (dpi) with P. infestans (Fig. 3 ), focusing mostly on new genes with no predictable function. As a positive control, the expression of the well-characterized elicitor ELI-INF1 (Kamoun et al. 1997) was monitored (PiE1) and, although transcripts were detected in cultured mycelia, mitochondrial (m)RNA levels were substantially higher during pathogenesis. This result is different from that of Kamoun and associates (1997), who reported that ELI-INF1 was downregulated during infection of potato, although they used a tomatospecialized isolate of $P$. infestans and, therefore, this difference may simply reflect the differences in plant host. It also is noteworthy that the rate of infection of potato was significantly slower than that observed in this current study; therefore, the dynamics of infection also might contribute to the differing expression patterns. In contrast with the abundance of ELI-INF1 transcript in vitro, all the transcripts tested that encoded unknown or hypothetical $P$. infestans-secreted proteins were detected only in infected leaves and not in in vitro-grown mycelia. The presence of plant mRNAs with sequence homology to PiE6 and PiE7 was indicated by crossreacting bands of a different size in noninfected or wounded leaves (Fig. 3); however, crosshybridizing bands were not seen by Southern analysis of tomato genomic DNA at the same hybridization stringency, suggesting a low level of sequence similarity.

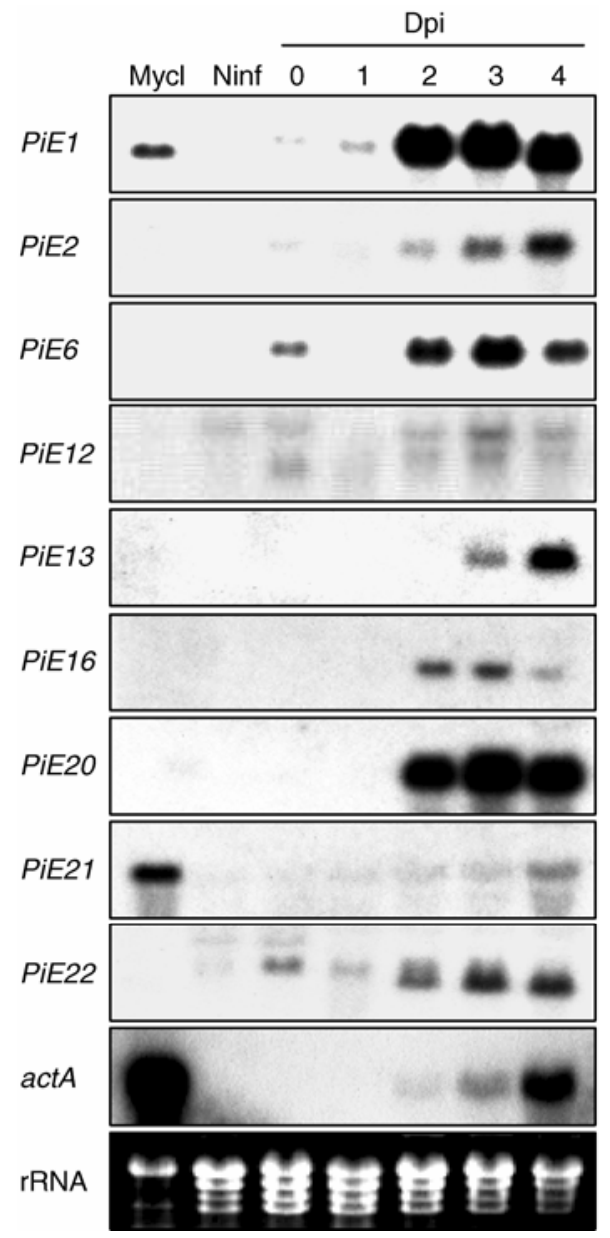

Fig. 4. RNA gel blot analysis of PiE clones expressed in tomato leaves over an infection time course. Total RNA $(20 \mu \mathrm{g})$ was isolated from axenically grown Phytophthora infestans mycelia (Mycl), noninfected tomato leaves (Ninf), or P. infestans-infected tomato leaves at $0,1,2,3$, and 4 days post inoculation (dpi). The blots were hybridized with the indicated PiE clones and a specific probe corresponding to P. infestans actin (actA). Ethidium bromide-stained rRNAs are shown to evaluate loading. 
Given that $P$. infestans is a hemibiotrophic organism, it is likely that different subsets of proteins are secreted into the host apoplast during the biotrophic or necrotrophic phases of the life cycle. To provide more insight into the temporal dynamics of the transcripts underlying the secretome, Northern blot analysis was used to monitor the abundance of a selection of the PiE mRNAs in a time course of infection from 1 to 4 dpi, again focusing on new hypothetical proteins (Fig. 4). The expression of a $P$. infestans actin gene (actA) used as a constitutively expressed control (Unkles et al. 1991) first was detected at 2 dpi and its abundance increased thereafter, providing an indication of the relative oomycete biomass. Most of the PiE genes were detected again only in infected tissues rather than in axenically grown mycelia, and they exhibited a range of expression patterns in terms of the timing of onset and maximal levels of transcript accumulation. Some genes, such as PiE2 and $P i E 13$, appeared to be expressed constitutively over the 4day time course, in that mRNA abundance paralleled the equivalent relative abundance of $a c t A$ mRNA. Others were expressed predominantly early during infection and showed a decrease in signal intensity on the Northern blot (e.g., PiE6 and $P i E 16)$, or a relative decrease in expression in proportion to the accumulation of hyphal biomass (e.g., PiE2O and PiE22), as judged by actA mRNA levels.

\section{Analysis of PiE12, PiE14, and PiE23 protein structure.}

To gain insight into putative functions for the unidentified PiE candidates, translated sequences were submitted to Motif Scan for prediction of functional domains. In addition to the N-terminal SP, PiE12, PiE14, and PiE23 were predicted to contain bipartite nuclear localization signals (NLSs) (data not shown).

A conserved host-targeting signal for translocation of proteins from Plasmodium spp. (malaria parasites) into host cells has been described recently (Hiller et al. 2004; Marti et al. 2004) and a similar motif, termed RXLR, is present in some secreted oomycete proteins (Armstrong et al. 2005; Rehmany et al. 2005). Recently, it has been reported that the Phytophthora RXLR motif indeed acts as a host-targeting signal in Plasmodium (Bhattacharjee et al. 2006). Visual inspection of the PiE12, PiE14, and PiE23 translations revealed an RXLR-like motif (where $\mathrm{X}$ is represented by Q/NLGAGGG) within 60 amino acids of the N-terminus, preceded by an SP and followed by NLSs, indicating that they represent an alternative form of the classical RXLR motif containing proteins. Homologous sequences of PiE12, PiE14, and PiE23 were obtained by searching the sequence contigs in Phytophthora sojae. The protein sequences of PiE12, PiE14, and PiE23 showed 65, 54, and 61\% homology to Ps_135684, Ps_135689, and Ps_135685, respectively, at the amino acid level. All the homologous sequences contained SPs, RXLR-like motifs, and predicted NLSs and were found to be clustered in a $20-\mathrm{kb}$ sequence contig in the genome of $P$. sojae, further suggesting that they may represent members of a gene family (Fig. 5).

\section{DISCUSSION}

The YST screen effectively targets both host and pathogen secretomes that are expressed in planta during pathogenesis.

The excellent progress that has been made recently in characterizing the TTSS-related secretomes of phytopathogenic bacteria has been achieved by coupling bioinformatic and high-throughput experimental approaches. For example, this has provided a near to complete coverage of the suites of effector proteins that are translocated into plant cells (Alfano and Collmer 2004; Chang et al. 2005). In contrast, far less is known about the secretomes of eukaryotic phytopathogens, notably fungi and oomycetes. In part, this may be because many of these organisms are obligate biotrophs and, therefore, more difficult to study, but it is striking that no coupled bioinformatic and high-throughput functional secretome screens analogous to the bacterial secretome studies have been described to date.

Several computational tools have been developed to predict the presence of N-terminal SPs that direct secreted proteins to the endoplasmic reticulum (ER) or Golgi-dependent, secretory pathway. For example, SignalP version 2.0 was used effectively to screen 1,000 P. infestans unigenes for secretory proteins (Torto et al. 2003). Computational prediction is an attractive approach, particularly in the context of large-scale evaluations, due to its relative ease and speed. However, as with all prediction-

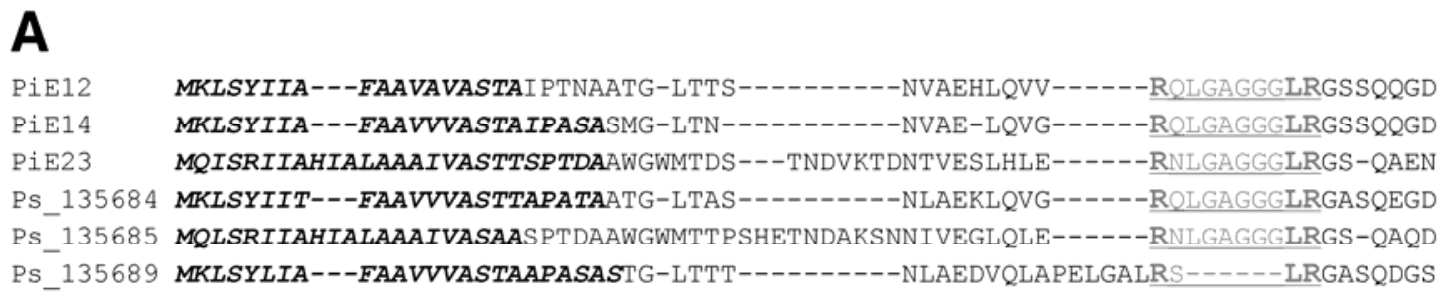

B

$20 \mathrm{~kb}$ sequence contig of $P$. sojae

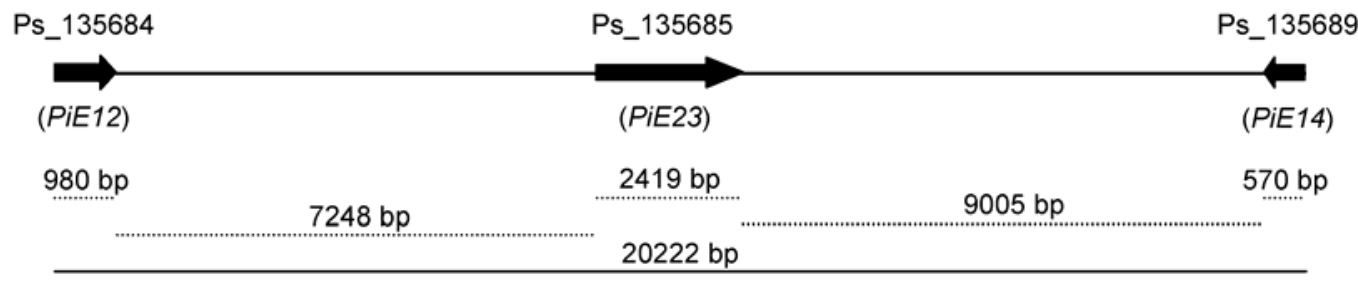

Fig. 5. Sequence alignment and genomic organization of PiE12, PiE14, and PiE23. A, Alignment of the N-terminal sequences of PiE12, PiE14, PiE23, and homologs from Phytophthora sojae (Ps). The N-terminal signal peptide is indicated in italics and the conserved, expanded RXLR-like motif is underlined. B, Clustering of homologs of PiE12, PiE14, and PiE23 in P. sojae. Arrows indicate homologs of PiE12, PiE14, and PiE23 and their orientations. A thin horizontal line represents a DNA sequence contig. 
based tools, there is a consistent error rate and both false positive and negative predictions should be expected; for example, Torto and associates (2003) reported a 17\% false positive error rate, which is not unusual for such programs. Therefore, as was illustrated with the bacterial secretome analyses, experimental screens provide an important complement to bioinformatic analyses by functionally validating or refuting the predictions.

The YST has been used to identify genes encoding secreted and plasma membrane-localized proteins from a range of eukaryotes (Lee et al. 2004), including a study of secreted plant proteins from $P$. parasitica-resistant tobacco leaves (Hugot et al. 2004). However, to date, no reports exist of its use to screen a library of pathogen-infected plant tissues. The objective of this study was to determine whether the YST screen represents an effective tool that ultimately could be used in combination with existing bioinfomatic approaches to examine the secretomes of both host and eukaryotic phytopathogen in planta during pathogenesis.

The efficiency of the YST approach is largely dependent on optimizing the proportion of heterologous cDNA sequences that can be generated in frame with invertase and on minimizing the background level of false positives. The new pYST three-vector system (Fig. 1B) was developed to address these criteria and resulted in significant improvements over the existing YST vector on both counts. Moreover, it was demonstrated that it could identify both oomycetous and fungalsecreted proteins (Fig. 1C). Another potential obstacle to this approach was the possibility that $P$. infestans sequences would not be detectable due to the high ratio of plant to oomycete biomass in the infected tissue; however, comparable numbers of plant and pathogen clones were identified (Tables 1 and 2). Because the majority of the PiE clones have homologs in the $P$. sojae and $P$. ramorum genomes (Table 1), it is likely that these three species secrete similar suites of proteins during infection of their respective hosts.

\section{Many $P$. infestans-secreted proteins are expressed only in planta and exhibit temporal differences in expression.}

Of the tomato SlE genes that were identified, half had some relationship with defense responses, including some of the wellstudied hydrolases that degrade the microbial hyphal walls or proteins involved in wall reinforcement. Others were annotated based on induction by wounding or an elicited response, but no known biochemical action. Interestingly, three clones (SIE9, SlE10, and SlE22) clearly had no predicted SP (Table 2) and, indeed, had entirely hydrophilic rather than characteristic hydrophobic N-termini (data not shown). One explanation for the identification of SP-less sequences is the existence of a "nonclassical" (ER or Golgi independent) secretory pathway, as has been described in mammals and a lower eukaryote (Nickel 2005). A recent review also cataloged more than 40 secreted but SP-less proteins in yeast (Nombela et al. 2006), presenting several alternative protein secretory routes. We are aware of no demonstrated examples in the literature of plant proteins that are secreted by nonclassical routes, although studies in our lab recently have demonstrated their existence (data not shown). This further emphasizes the value of the YST screen, because it potentially identifies all secreted proteins, as opposed to computational prediction that currently is adequate only for proteins with classical SPs. Nevertheless, we cannot exclude the possibility that the YST screen yielded false positives.

Most of the PiE genes had no predictable function, but those that were annotated included known or putative effectors, such as elicitin (PiE1), cutinase (PiE18), and a protein with homology to a necrosis-inducing phytotoxin (PiE19). An intriguing exception was PiE16, which has sequence homology with a mollusk protein pheromone. Sexual reproduction in $P$. infestans involves the interaction between two distinct mating types, which is mediated by attractants and, although a recently identified Phytophthora mating hormone was nonproteinaceous (Qi et al. 2005), it would be interesting to see whether PiE16 has a related function. It was noted that, of the $23 \mathrm{PiE}$ clones, only three (PiE1, PiE5, and PiE6) were previously reported in the Torto and associates (2003) bioinfomatics analysis.

Northern blot analysis of the PiE clones with no known function revealed that most were expressed only in planta during pathogenesis. Moreover, the temporal variation in transcript expression patterns of the PiE genes (Fig. 4) supports the idea that different subsets of proteins are secreted into the plant apoplast at various times during infection; in this case, perhaps in association with distinct phases of $P$. infestans biotrophy or necrotrophy. These data further suggest that a YST screen of infected plant tissues is a valuable means to identify the crucial subset of effectors and pathogenicity determinants that are expressed in planta, as opposed to computational prediction based on EST collections from cultured organisms, particularly for "nonmodel" phytopathogens that are not currently targeted by large-scale sequencing projects. This certainly is also the case for obligate biotrophs that cannot be grown axenically and microbial symbionts, such as mycorrhizal fungi.

\section{MATERIALS AND METHODS}

\section{$P$. infestans strain, culture conditions, and inoculum production.}

The tomato-specialized $P$. infestans isolate US970001 (ATCC \#MYA-2350), a member of the US-17 clonal lineage (Smart et al. 2003), was cultured in rye B agar (Caten and Jinks 1968 ) at $15^{\circ} \mathrm{C}$ with $18 \mathrm{~h}$ of light per day. To generate zoospores for subsequent inoculation of tomato plants, detached tomato leaflets were inoculated with a $P$. infestans sporangial suspension and incubated in moist chambers for 5 days as described in Smart and associates (1998). Sporangia then were collected in water, diluted to 20,000 sporangia $\mathrm{ml}^{-1}$, and incubated at $4^{\circ} \mathrm{C}$ for at least $2 \mathrm{~h}$ to induce zoospore release.

\section{Plant material and infection by $\boldsymbol{P}$. infestans.}

Tomato (S. lycopersicum cv. Rio Grande) plants were grown in a greenhouse at Cornell under conditions of $16 \mathrm{~h}$ of light and $8 \mathrm{~h}$ of dark. Attached leaves from 4-week-old plants were spray inoculated with $P$. infestans zoospore suspensions in a humid chamber at $15^{\circ} \mathrm{C}$ with $16 \mathrm{~h}$ of light and $12 \mathrm{~h}$ at $100 \%$ relative humidity, as described by Smart and associates (2003). Symptoms were monitored daily and leaves were harvested at the indicated times for RNA extraction and cDNA library construction. For the wounded leaf material, young attached leaves were stabbed with a block of needles and left on the plant for $6 \mathrm{~h}$ before harvesting. All plant tissue was frozen in liquid nitrogen immediately after collection and stored at $-80^{\circ} \mathrm{C}$.

\section{Construction}

of the pYST yeast signal sequence trap library vectors.

The pYST-0, pYST-1, and pYST-2 vectors for construction of YST libraries were derived from the pSMASH plasmid (Goo et al. 1999) (a generous gift from Dr. O.K. Park), using PCR to generate variants of the pYST vector that allow the cloning of cDNA inserts in all three reading frames in frame with the $s u c 2$ invertase gene. The PCR primers used were suc2-5 (5'-GCGCGCGCGGCCGCC(0-2)TTGGATAAAAGG TACCCAAACGAAACTAGC-3') and suc2-3 (5'-CATGTTTG GTGATATCATCTCCGATGAAGCCTCCGTTATCCCA-3'). The PCR products were subcloned as NotI-EcoRV fragments into pSMASH that previously had been digested with NotI and EcoRV and sequenced. The suc2-5 primer was designed such 
that the first two amino acid $\left(\mathrm{Met}^{21}\right.$ and $\mathrm{Thr}^{22}$ ) of the suc2 mature protein were eliminated, unlike the original pSMASH vector.

\section{Construction of a $P$. infestans-infected tomato leaf cDNA library and control plasmid DNAs.}

To create the $P$. infestans-infected tomato leaf YST cDNA library, total RNA was isolated as described by Rose and associates (1996) from a pooled sample of tomato leaves 1, 2, 3, and 4 dpi with $P$. infestans. Poly(A) mRNA was purified from total RNA using an Oligotex mRNA Midi Kit (Qiagen, Valencia, CA, U.S.A.) according to the manufacturer's instructions. The first-strand cDNAs were synthesized from mRNA using the N6-NotI primer (5'-GAGAGAGAGAGAGAGAACCGCG CGGCCGCCNNNNNN-3'; the underlined bases correspond to the NotI site) and a cDNA Synthesis Kit (Stratagene, La Jolla, CA, U.S.A.). After second-strand synthesis, the cDNAs were prepared for unidirectional cloning by ligation with EcoRI adapters according to the manufacturer's instructions (Stratagene), followed by NotI digestion. The cDNA fragments $(0.3$ to $1.0 \mathrm{~kb})$ were recovered following agarose gel electrophoresis with a Gel Extraction Kit (Qiagen) and ligated to a mixture of the three vectors (pYST-0, pYST-1, and pYST-2) that previously had been digested with EcoRI and NotI. Ligations were transformed into electrocompetent TOP10 Escherichia coli (Invitrogen, Carlsbad, CA, U.S.A.) by electroporation and the transformants selected on an ampicillin-containing medium. cDNA library plasmid DNA was purified using Perfectprep Midi spin columns (Brinkmann Instruments Inc., Westbury, NY, U.S.A.) from a pool of the transformed bacterial colonies.

To generate control plasmids to test the YST system with genes encoding known secreted proteins from eukaryotic plant pathogens, cDNAs encoding the open reading frames of $P$. sojae PsGIP1 (Rose et al. 2002) and C. carbonum CcEXG2 (Kim et al. 2001), with or without the region corresponding to the predicted SP, were amplified by PCR from genomic DNA of $P$. sojae (race 1) or the CcEXG2 cDNA (a generous gift from Dr. J. Walton). The following gene-specific primers were used that incorporated EcoRI and NotI restriction sites for subsequent cloning into the pYST-0 or pYST-1 vectors for PsGIP1 or $C c E X G 2$, respectively: Ps $G I P 1^{1-257} 5^{\prime}$-GCGCGCG AATTCATGAAGGTTTTCCCCGCC-3' and 5'-GCGCGCGC GGCCGCCTTCTTGATGACAGAGTT-3'; PsGIP1 $1^{22-257} \quad\left(5^{\prime}-\right.$ GCGCGCGAATTCATGGAACACGTGCAGCGCTCC-3' and 5'-GCGCGCGCGGCCGCCTTCTTGATGACAGAGTT-3'; CcEXG2 $2^{1-422} 5^{\prime}$-GCGCGCGAATTCATGATTCTCACCAAAC TC-3' and 5'-GCGCGCGCGGCCGCGCAGGCAGCGCCAA ACTT-3'; and CcEXG2 $2^{19-422} 5^{\prime}$-GCGCGCGAATTCATGGCTC CTGCCCAGCAGAAG-3' and 5'-GCGCGCGCGGCCGCGC AGGCAGCGCCAAACTT-3'.

The PCR conditions were $94^{\circ} \mathrm{C}$ for $3 \mathrm{~min} ; 35$ cycles of $94^{\circ} \mathrm{C}$ for $30 \mathrm{~s}, 55^{\circ} \mathrm{C}$ for $60 \mathrm{~s}$, and $72^{\circ} \mathrm{C}$ for $90 \mathrm{~s}$; and finally $72^{\circ} \mathrm{C}$ for $10 \mathrm{~min}$. PCR products were digested with EcoRI and NotI and cloned into the appropriate vectors, and the resulting plasmids PsGIP1 $1^{1-257}:$ suc2, PsGIP1 $1^{22-257}:$ suc2, CcEXG2 $2^{1-422}: s u c 2$, or $\mathrm{Cc} E X G 2^{19-422}: s u c 2$ were sequenced to confirm an in-frame fusion with suc2.

\section{Yeast transformation and selection.}

cDNA library plasmids were transformed into the yeast (Saccharomyces cerevisiae) strain DBY 2445 (Mat $\alpha$, suc2 $\Delta$ 9, lys2-801, ura3-52, ade2-101) (Goo et al. 1999) (a generous gift from Dr. O.K. Park) using the YEASTMAKER Yeast Transformation System 2 (BD Biosciences, San Jose, CA, U.S.A.). Yeast transformants appeared on a sucrose agar plate ( $1 \%$ yeast extract, $2 \%$ peptone, $2 \%$ sucrose, and $2 \%$ agar) after incubation at $30^{\circ} \mathrm{C}$ for 6 to 10 days. The colonies were restreaked on sucrose agar plates, and plasmid DNA was electroporated into XL1Blue E. coli cells and subsequently sequenced using a primer corresponding to $\mathrm{ADH} 1$ promoter of the pYST vectors ( $5^{\prime}$-TCCTCGTCATTGTTCTCGTTCC-3'). To eliminate redundant YST clones, the first set of approximately 50 unique cDNA inserts, obtained by digesting a mixed pool of plasmid DNAs with EcoRI and NotI, were gel purified, labeled with $\left[\alpha-{ }^{32} \mathrm{P}\right]-\mathrm{dCTP}$, and used to probe a DNA gel blot of the inserts from the next set of positive yeast colonies, obtained by colony PCR using the primers Y-5 (5'-TCCTCGTCA TTGTTCTCGTTCC-3') and Y-3 (5'-CCTTTTATCCAAGCGG CC-3'). Colony PCR was performed using a program of 35 cycles at $94^{\circ} \mathrm{C}$ for $30 \mathrm{~s}, 55^{\circ} \mathrm{C}$ for $45 \mathrm{~s}$, and $72^{\circ} \mathrm{C}$ for $60 \mathrm{~s}$, with an initial denaturing step at $94^{\circ} \mathrm{C}$ for $3 \mathrm{~min}$ and a final extension at $72^{\circ} \mathrm{C}$ for $10 \mathrm{~min}$. Hybridization and washing conditions were as described below for the genomic DNA gel blot analysis. Plasmid DNA from each nonhybridized individual yeast colony was recovered for the second round of sequencing analysis. To eliminate the redundant clones completely from all the positive yeast colonies, several rounds of the screening were performed as described above. In all cases, cDNAs encoding proteins lacking a predicted SP were retransformed into the yeast strain to confirm the ability to allow growth on a sucrose agar plate.

\section{Sequence analysis.}

Sequences of the PiE and SlE clones were used to search GenBank, the TIGR Tomato Gene Index, the Solanaceae Genomics Network, and the Phytophthora Functional Genomics Database. Signal peptides and putative cleavage sites were predicted by SignalP (version 3.0). Sequences were aligned using ClustalW software and adjusted manually. Prediction of nuclear localization signals was performed using MotifScan. $P$. sojae and $P$. ramorum homologs of PiE12 were identified in the microbial genome database and subjected to motif analysis as described above.

\section{DNA gel blot analysis.}

Genomic DNA was isolated from $P$. infestans mycelia and from young tomato leaves as described previously (Rose et al. 1997, 2002). The genomic DNA samples were digested with the indicated restriction enzymes, separated by electrophoresis on a $0.8 \%$ agarose gel, denatured, and blotted onto nylon membranes (Amersham Biosciences, Piscataway, NJ, U.S.A.). Full-length cDNA probes were labeled with $\left[\alpha-{ }^{32} \mathrm{P}\right]$-dCTP using the Ready-To-Go DNA Labeling Kit (Amersham Biosciences) and purified with ProbeQuant G-50 Micro Columns (Amersham Biosciences). Hybridization and washing procedures were as described by Catalá and associates (2001) with a final wash in $0.1 \times \mathrm{SSC}(1 \times \mathrm{SSC}$ is $0.15 \mathrm{M} \mathrm{NaCl}$ plus $0.015 \mathrm{M}$ sodium citrate) and $0.1 \%$ sodium dodecyl sulfate (SDS) at $65^{\circ} \mathrm{C}$ for $1 \mathrm{~h}$. After washing, membranes were exposed to X-ray film.

\section{RNA gel blot analysis.}

Total RNA was isolated as described previously (Rose et al. 1996) from $P$. infestans mycelia and from infected tomato leaves $1,2,3$, and 4 dpi with $P$. infestans. Aliquots (20 $\mu \mathrm{g}$ per lane) were fractionated on $1.0 \%$ (wt/vol) agarose $/ 10 \%$ (vol/vol) formaldehyde denaturing gels, visualized with ethidium bromide to confirm equal loading and transferred onto Hybond $\mathrm{N}$ nylon membranes (Amersham Biosciences). Hybridization was performed as described by Catalá and associates (2001) and washes were carried out twice in $2 \times$ $\mathrm{SSC} / 0.1 \% \mathrm{SDS}$ at $65^{\circ} \mathrm{C}$ and twice in $0.1 \times \mathrm{SSC} / 0.1 \% \mathrm{SDS}$ at $65^{\circ} \mathrm{C}$ for $15 \mathrm{~min}$ each. 


\section{ACKNOWLEDGMENTS}

The authors would like to thank O. K. Park (Graduate School of Biotechnology, Korea University, Seoul, Korea) for the generous gift of the pSMASH plasmid and the yeast strain DBY $\alpha 2445$ and J. Walton (MSUDOE Plant Research Laboratory, Michigan State University) for providing the $C$. carbonum $C c E X G 2$ cDNA. We are also grateful for the technical assistance and advice provided by C. Smart, M. Saladié, B. Fry, C. Catalá (Cornell University), and S. Kamoun (Ohio State University). This work was supported by a grant from Korea Science and Engineering Foundation Ministry of Science and Technology (KOSEF/MOST) to S. J. Lee, B.-S. Kim, and B.-D. Kim of the Center for Plant Molecular Genetics and Breeding Research, and from the United States Department of Agriculture IFAFS-MGET program (2001-52014-11484) to B. S. Kelley. C. M. B. Damasceno was supported by a student fellowship from the Conselho Nacional de Desenvolvimento Científico e Tecnológico, Brazil and B. St. John by a National Science Foundation (NSF) Plant Genome outreach fellowship (DBI-0076892). Additional funding was provided to J. K. C. Rose by the NSF award DBI-0431335 and by a grant from New York State Office of Science, Technology and Academic Research (NYSTAR).

\section{LITERATURE CITED}

Abramovitch, R. B., and Martin, G. B. 2004. Strategies used by bacterial pathogens to suppress plant defenses. Curr. Opin. Plant Biol. 7:356-364.

Alfano, J. R., and Collmer, A. 2004. Type III secretion system effector proteins: Double agents in bacterial disease and plant defense. Annu. Rev. Phytopathol. 42:385-414.

Armstrong M. R., Whisson S. C., Pritchard L., Bos J. I., Venter E., Avrova A. O., Rehmany A. P., Bohme U., Brooks K., Cherevach I., Hamlin N., White B., Fraser A., Lord A., Quail M. A., Churcher C., Hall N., Berriman M., Huang S., Kamoun S., Beynon J. L., and Birch P. R. 2005. An ancestral oomycete locus contains late blight avirulence gene Avr $3 a$, encoding a protein that is recognized in the host cytoplasm. Proc. Natl. Acad. Sci. U.S.A. 102:7766-7771.

Bendtsen, J. D., Nielsen, H., von Heijne, G., and Brunak, S. 2004. Improved prediction of signal peptides: SignalP 3.0. J. Mol. Biol. 340:783-795.

Bhattacharjee, S., Hiller, N. L., Liolios, K., Win, J., Kanneganti, T.-D., Young, C., Kamoun, S., and Haldar, K. 2006. The malarial host-targeting signal is conserved in the Irish potato famine pathogen. PLoS Pathog. 2:e50.

Bos, J. I. B., Armstrong, M., Whisson, S. C., Torto, T. A., Ochwo, M., Birch, P. R. J., and Kamoun, S. 2003. Intraspecific comparative genomics to identify avirulence genes from Phytophthora. New Phytol. 159:63-72.

Catalá, C., Rose, J. K. C, York, W. S., Albersheim, P., Darvill, A. G., and Bennett, A. B. 2001. Characterization of a tomato xyloglucan endotransglycosylase gene that is down-regulated by auxin in etiolated hypocotyls. Plant Physiol. 127:1180-1192.

Catanzariti, A.-M., Dodds, P. D., Lawrence, G. J., Ayliffe, A M. A., and Ellis, J. G. 2006. Haustorially expressed secreted proteins form flax rust are highly enriched for avirulence elicitors. Plant Cell 18:243-256.

Caten, C. E., and Jinks, J. L. 1968. Spontaneous variability of single isolates of Phytophthora infestans. I. Cultural variation. Can. J. Bot. 46:329-348.

Chang, J. H., Goel, A. K., Grant, S. R. and Dangl, J. L. 2004. Wake of the flood: Ascribing functions to the wave of type III effector proteins of phytopathogenic bacteria. Curr. Opin. Microbiol. 7:11-18.

Chang, J. H., Urbach, J. M., Law, T. F., Arnold, L. W., Hu, A., Gombar, S., Grant, S. R., Ausubel, F. M., and Dangl, J. 2005. A high-throughput, near-saturating screen for type III effector genes from Pseudomonas syringae. Proc. Natl. Acad. Sci. U.S.A. 102:2549-2554.

Cummins, S. F., Nichols, A. E., Amare, A., Hummon, A. B., Sweedler, J. V., and Nagle, G. T. 2004. Characterization of Aplysia enticin and temptin, two novel water-borne protein pheromones that act in concert with attractin to stimulate mate attraction. J. Biol. Chem. 279:25614-25622.

Dangl, J. L., and Jones, J. D. G. 2001. Plant pathogens and integrated defence responses to infection. Nature 411:826-833.

Goo, J. H., Park, A. R., Park, W. J., and Park, O. K. 1999. Selection of Arabidopsis genes encoding secreted and plasma membrane proteins. Plant Mol. Biol. 41:415-423.

Greenberg, J. T., and Vinatzer, B. A. 2003. Identifying type III effectors of plant pathogens and analyzing their interactions with plant cells. Curr. Opin. Microbiol. 6:20-28.

Guttman, D. S., Vinatzer, B. A., Sarkar, S. F., Ranall, M. V., Kettler, G., and Greenberg, J. T. 2002. A functional screen for the type III (Hrp) secretome of the plant pathogen Pseudomonas syringae. Science 295:1722-1726.
Hiller, N. L., Bhattacharjee, S., van Ooij, C., Liolios, K., Harrison, T., Lopez-Estrano, C., and Haldar, K. 2004. A host-targeting signal in virulence proteins reveals a secretome in malarial infection. Science 306:1934-1937.

Hugot, K., Riviere, M. P., Moreilhon, C., Dayem, M. A., Cozzitorto, J., Arbiol, G., Barbry, P., Weiss, C., and Galiana, E. 2004. Coordinated regulation of genes for secretion in tobacco at late developmental stages: Association with resistance against oomycetes. Plant Physiol. 134:858-870.

Huitema, E., Bos, J. I. B., Tian, M., Win, J., Waugh, M. E., and Kamoun, S. 2004. Linking sequence to phenotype in Phytophthora-plant interactions. Trends Microbiol. 12:193-200.

Jacobs, K. A., Collins-Racie, L. A., Colbert, M., Duckett, M., Golden-Fleet, M., Kelleher, K., Kriz, R., La Vallie, E. R., Merberg, D., Spaulding, V., Stover, J., Williamson, M. J., and McCoy, J. M. 1997. A genetic selection for isolating cDNAs encoding secreted proteins. Gene 198:289-296.

Jiang, R. H. Y., Tyler, B. M., Whisson, S. C., Hardham, A. R., and Govers, F. 2006. Ancient origin of elicitin gene clusters in Phytophthora genomes. Mol. Biol. Evol. 23:338-351.

Kamoun, S., vanWest, P., deJong, A. J., deGroot, K. E., Vleeshouwers, V. G. A. A., and Govers, F. 1997. A gene encoding a protein elicitor of Phytophthora infestans is down-regulated during infection of potato. Mol. Plant-Microbe Interact. 10:13-20.

Kim, H., Ahn, J. H., Gorlach, J. M, Caprari, C., Scott-Craig, J. S., and Walton, J. D. 2001. Mutational analysis of beta-glucanase genes from the plant-pathogenic fungus Cochliobolus carbonum. Mol. PlantMicrobe Interact. 14:1436-1443.

Lee, S.-J., Saravanan, R. S., Damasceno, C. M. B., Yamane, H., Kim, B.D., and Rose, J. K. C. 2004. Digging deeper into the plant cell wall proteome. Plant Physiol. Biochem. 42:979-988.

Liu, Z., Bos, J. I., Armstrong, M., Whisson, S. C., da Cunha, L., TortoAlalibo, T., Win, J., Avrova, A. O., Wright, F., Birch, P. R., and Kamoun, S. 2005. Patterns of diversifying selection in the phytotoxin-like $s c r 74$ gene family of Phytophthora infestans. Mol. Biol. Evol. 22:659-672.

Marti, M., Good, R. T., Rug, M., Knuepfer, E., and Cowman, A. F. 2004. Targeting malaria virulence and remodeling proteins to the host erythrocyte. Science 306:1930-1933.

Nickel, W. 2005. Unconventional secretory routes: Direct protein export across the plasma membrane of mammalian cells. Traffic 6:607-614.

Nombela, C., Gil, C., and Chaffin, W. L. 2006. Non-conventional protein secretion in yeast. Trends Microbiol. 14:15-21.

Orsomando, G., Lorenzi, M., Raffaelli, N., Dalla Rizza, M., Mezzetti, B., and Ruggieri, S. 2001. Phytotoxic protein PcF, purification, characterization, and cDNA sequencing of a novel hydroxyproline-containing factor secreted by the strawberry pathogen Phytophthora cactorum. J. Biol. Chem. 276:21578-21584.

Qi, J., Asano, T., Jinno, M., Matsui, K., Atsumi, K., Sakagami, Y., and Ojika, M. 2005. Characterization of a Phytophthora mating hormone. Science 309:1828.

Qutob, D, Hraber, P. T., Sobral, B. W., and Gijzen, M. 2000. Comparative analysis of expressed sequences in Phytophthora sojae. Plant Physiol. 123:243-54.

Rast, D. M., Baumgartner, D., Mayer, C., and Hollenstein, G. O. 2003. Cell wall-associated enzymes in fungi. Phytochemistry 64:339-366.

Rehmany, A. P., Gordon, A., Rose, L. E., Allen, R. L., Armstrong, M. R., Whisson, S. C., Kamoun, S., Tyler, B. M., Birch, P. R. J., and Beynon, J. L. 2005. Differential recognition of highly divergent downy mildew avirulence gene alleles by $R P P 1$ resistance genes from two Arabidopsis lines. Plant Cell 17:1839-1850.

Rep, M. 2005. Small proteins of plant-pathogenic fungi secreted during host colonization. FEMS (Fed. Eur. Microbiol. Soc.) Microbiol. Lett. 253:19-27.

Rose, J. K. C., Brummell, D. A., and Bennett, A. B. 1996. Two divergent xyloglucan endotransglycosylases exhibit mutually exclusive patterns of expression in nasturtium. Plant Physiol. 110:493-499.

Rose, J. K. C., Ham, K.-S., Darvill, A. G., and Albersheim, P. 2002. Molecular cloning and characterization of glucanase inhibitor proteins: Coevolution of a counterdefense mechanism by plant pathogens. Plant Cell 14:1329-1345.

Rose, J. K. C., Lee, H. H., and Bennett, A. B. 1997. Expression of a divergent expansin gene is fruit-specific and ripening-regulated. Proc. Natl. Acad. Sci. U.S.A. 94:5955-5960.

Smart, C. D., Myers, K. L., Restrepo, S., Martin, G. B., and Fry, W. E. 2003. Partial resistance in tomato to Phytophthora infestans is not dependent upon ethylene, jasmonic acid or salicylic acid signaling pathways. Mol. Plant-Microbe Interact. 16:141-148.

Smart, C. D., Willmann, M. R., Mayton, H., Mizubuti, E. S. G., Sandrock, R. W., Muldoon, A. E., and Fry, W. E. 1998. Self-fertility in two clonal lineages of Phytophthora infestans. Fungal Genet. Biol. 25:134-142.

Takemoto, D., Yoshioka, H., Doke, N., and Kawakita, K. 2003. Disease stress-inducible genes of tobacco: Expression profile of elicitor-re- 
sponsive genes isolated by subtractive hybridization. Physiol. Plant. 118:545-553.

Torto, T. A., Li, S., Styer, A., Huitema, E., Testa, A., Gow, N. A. R., van West, P., and Kamoun, S. 2003. EST mining and functional expression assays identify extracellular effector proteins from the plant pathogen Phytophthora. Genome Res. 13:1675-1685.

Unkles, S. E., Moon, R. P., Hawkins, A. R., Duncan, J. M., and Kinghorn, J. R. 1991. Actin in the oomycetous fungus Phytophthora infestans is the product of several genes. Gene 100:105-112.

van der Hoorn, R. A. L., and Jones, J. D. G. 2004. The plant proteolytic machinery and its role in defence. Curr. Opin. Plant Biol. 7:400-407.

Vorwerk, S., Somerville, S., and Somerville, C. 2004. The role of plant cell wall polysaccharide composition in disease resistance. Trends Plant Sci. 9:203-209.
AUTHOR-RECOMMENDED INTERNET RESOURCES

Cornell University's Solanaceae Genomics Network: soldb.cit.cornell.edu or www.sgn.cornell.edu

European Bioinformatics Institute (EBI) ClustalW website: www.ebi.ac.uk/clustalw

Joint Genome Institute (JGI) Eukaryotic Genomics website: genome.jgi-psf.org/euk_home.html

National Center for Biotechnology Information (NCBI) GenBank: www.ncbi.nlm.nih.gov

Phytophthora Functional Genomics database: www.pfgd.org

Prosite's Motif Scan: myhits.isb-sib.ch/cgi-bin/motif_scan

SignalP server: www.cbs.dtu.dk/services/SignalP

TIGR Tomato Gene Index: www.tigr.org/tdb/tgi/lgi 